\title{
Influência da argila vermiculita brasileira na biodegradação de filmes de PHB
}

\section{Influence of brazilian vermiculite clay in biodegradation of PHB films}

\author{
Rayson de Jesus Araújo ${ }^{1}$, Isaias Damasceno da Conceição ${ }^{1}$, Laura Hecker de Carvalho², \\ Tatianny Soares Alves ${ }^{3}$ e Renata Barbosa ${ }^{3 *}$
}

\author{
${ }^{1}$ Departamento de Engenharia Mecânica, Universidade Federal do Piauí - UFPI, Teresina, PI, Brasil \\ 2Programa de Pós-graduação em Ciência e Engenharia de Materiais, Universidade Federal de Campina \\ Grande - UFCG, Campina Grande, PB, Brasil \\ ${ }^{3}$ Programa de Pós-graduação em Ciência dos Materiais, Universidade Federal do Piauí - UFPI, \\ Campus Universitário Ministro Petrônio Portella, Teresina, PI, Brasil
}

*rrenatabarbosa@yahoo.com

\begin{abstract}
Resumo
Este trabalho teve como objetivo preparar e avaliar filmes PHB/vermiculita natural e modificada nas quantidades em peso de $1 \%, 3 \%$ e $6 \%$ através dos métodos intercalação por fusão e intercalação por solução. Os bionanocompósitos obtidos pelo método intercalação por fusão foram preparados em uma extrusora monorosca e posteriormente os filmes foram moldados via compressão. Na preparação dos filmes por solução, os sistemas foram submetidos à agitação e aquecimento a $80^{\circ} \mathrm{C}$. Os sistemas foram avaliados por difração de raios-X e o comportamento de biodegradação foi avaliado de acordo com a norma ASTM G 160-03. O acompanhamento da biodegradação foi realizado por meio de inspeção visual e perda de massa. Observou-se que o percentual de argila e o método de obtenção dos filmes influenciaram na estrutura formada e na biodegradação dos sistemas.
\end{abstract}

Palavras-chave: polímeros biodegradáveis, argila vermiculita, ensaio de biodegradação, bionanocompósitos.

\begin{abstract}
This study aimed to prepare and to evaluate natural and modified PHB /vermiculite films in quantities of $1 \%, 3 \%$ and $6 \%$ by weight, through melt intercalation and solution intercalation methods. The bionanocomposites in the form of thin films, obtained by the melt intercalation method, were prepared in a single screw extruder and then molded via compression. In the preparation of the films by solution method, the systems were stirred and heated at $80{ }^{\circ} \mathrm{C}$ in the presence of the chloroform solvent. The systems were evaluated by X-ray diffraction and the degradation behavior was evaluated according to ASTM G 160-03, by visual inspection and through weight loss. It was observed that the percentage of clay and the method of obtaining of the films influenced in the structure and biodegradability of the systems.
\end{abstract}

Keywords: biodegradable polymers, vermiculite clay, biodegradation test, bionanocomposites.

\section{Introdução}

O uso de plásticos sintéticos convencionais, polímeros derivados do petróleo, como material de embalagem é elevado, principalmente por sua disponibilidade, baixo custo e características funcionais, destacando-se as boas propriedades mecânicas, barreira aos gases e compostos aromáticos, e a facilidade de selagem térmica. No entanto, apesar das grandes vantagens dos plásticos sintéticos, seu uso crescente gera preocupação devido a problemas de contaminação ambiental decorrentes do descarte, uma vez que não são biodegradáveis e sua reciclagem consome grandes quantidades de energia térmica. $\mathrm{O}$ interesse de manter, ou melhorar, a qualidade dos produtos embalados e, ao mesmo tempo, reduzir o desperdício de embalagens, têm

incentivado a exploração de novos materiais de embalagens, como os filmes biodegradáveis formados de matérias-primas oriundas de recursos renováveis ${ }^{[1]}$.

$\mathrm{O}$ uso de polímeros biodegradáveis destinados em especial para aplicações em embalagens alimentícias está aumentando consideravelmente, no intuito de reduzir a utilização de recursos não-renováveis e, com isso, impedir também o acúmulo de resíduos plásticos no meio ambiente. Em paralelo, a indústria de embalagens vem exigindo o desenvolvimento de novas misturas/formulações, processamentos mais simples e diminuição de custo no uso de suas tecnologias ${ }^{[2]}$. Ao mesmo tempo dessas preocupações 
anteriormente citadas, o consumidor também exige: alta qualidade do alimento e data de validade mais prolongada, além disso, que a embalagem seja transparente para facilitar a visualização do alimento e resistente quanto a contaminação, umidade e oxidação ${ }^{[3]}$.

Os problemas decorrentes da poluição ambiental gerada pelo lixo plástico têm levado a comunidade científica a refletir sobre possíveis alternativas para o problema. Para o gerenciamento da enorme quantidade de lixo plástico produzido pela sociedade, a biodegradação é uma das alternativas que tem sido proposta e atualmente têm sido alçada a uma posição de destaque. A biodegradação consiste na degradação dos materiais poliméricos através da ação de organismos vivos ${ }^{[4]}$.

A American Society for Testingand Materials (ASTM) tem proposto vários métodos de análise e acompanhamento da biodegradação dos polímeros, que consistem em ensaios de biodegradabilidade em exposição a microrganismos por meio de compostos em ambientes aeróbicos e anaeróbicos. Dentre os métodos propostos pela ASTM se destaca a norma ASTM G $160-03^{[5]}$ que avalia a susceptibilidade microbiológica de um material não metálico quando em contato com o ambiente natural do solo, e assim, o presente trabalho fundamenta-se nesta norma para a realização da avaliação da biodegradação dos filmes biodegradáveis.

O interesse pelos polímeros biodegradáveis na área de pesquisas científicas manifesta-se pelas propriedades semelhantes que eles possuem em relação aos plásticos convencionais ${ }^{[6]}$. Assim, existem vários tipos de polímeros biodegradáveis, destacando-se principalmente, o Polihidroxibutirato (PHB) que é produzido naturalmente por bactérias a partir de fontes renováveis de energia, e são biodegradados em alguns meses por uma enorme quantidade de bactérias e fungos presentes na natureza ${ }^{[7]}$. Porém, por ser produzido por um processo de fermentação bacteriana, ainda é um processo relativamente $\operatorname{caro}^{[8]}$.

Os altos custos para produzir o polihidroxibutirato têm levado as empresas a incorporar materiais inorgânicos, como certos tipos de argilas no processo de fabricação, a fim de reduzir esses custos e para melhorar as propriedades do produto final, tornando-o mais competitivo em relação aos plásticos convencionais. Dentre as argilas utilizadas, a vermiculita se destaca por causa de sua propriedade de expandir-se a elevadas temperaturas, tornando-se um material leve, além de que, pode ser encontrada em abundância em algumas regiões no Brasil ${ }^{[9]}$.

O uso de nanocompósitos pode ser uma boa solução tecnológica para melhorar as deficientes propriedades dos materiais para fins de embalagens. As nanopartículas desejadas devem ser recicladas e biodegradáveis ${ }^{[3]}$. A incorporação da argila vermiculita ao polihidroxibutirato (PHB) proporciona a formação de bionanocompósitos poliméricos. Em geral, os materiais inorgânicos não apresentam uma boa interação com os polímeros orgânicos, assim faz-se necessária a modificação desses materiais inorgânicos, tornando-os organofílicos, para que haja uma melhor interação polímero/argila e dessa maneira melhorar as propriedades dos bionanocompósitos poliméricos ${ }^{[10]}$.

Os bionanocompósitos poliméricos podem ser obtidos através de quatro métodos: Polimerização in situ, intercalação por solução, método sol-gel e intercalação no estado fundido ${ }^{[11]}$. O método intercalação no estado fundido, é um método rápido utilizado para obtenção dos nanocompósitos, não necessita da utilização de solventes nocivos ao meio ambiente e à saúde, e o processamento ocorre com o mínimo prejuízo para as propriedades do PHB. No método intercalação por solução permite observar o grau de inchamento da argila num determinado solvente.

Levando em consideração os fatores acima expostos, este artigo tem como objetivo principal avaliar a biodegradação de filmes biodegradáveis PHB/argila vermiculita natural e modificada de acordo com a norma ASTM G 160-03. Os filmes foram obtidos pelos métodos intercalação por fusão e intercalação por solução e, posteriormente, caracterizados por difração de raios-X. O acompanhamento da biodegradação foi realizado por inspeção visual e por perda de massa.

\section{Experimental}

\subsection{Materiais}

O Polihidroxibutirato (PHB) foi fornecido pela PHB Industrial S/A, São Paulo; a argila pela Mineração Pedra Lavrada, Santa Luzia - PB e o sal de amônio utilizado foi o Praepagen WB ${ }^{\circledR}$ (cloreto de estearildimetil amônio), fornecido pela Clariant, Recife - PE. O clorofórmio utilizado no método intercalação por solução foi fabricado pela Dinâmica Química Contemporânea Ltda. O solo utilizado no teste de biodegradação foi um composto orgânico comercial a base de esterco bovino e de galinha.

\subsection{Processo de organofilização da argila}

O processo de organofilização consistiu no preparo de dispersões contendo concentrações de água destilada, argila e o sal de amônio, conforme métodos propostos por Barbosa et al. ${ }^{[12]}$ e Mesquita ${ }^{[13]}$

\subsection{Método intercalação por fusão}

Os sistemas foram preparados em uma extrusora monorosca modelo AX-16 da AX Plásticos sob temperaturas variando entre $160^{\circ} \mathrm{C}, 165^{\circ} \mathrm{C}$ e $175^{\circ} \mathrm{C}$, da primeira para a terceira zona, respectivamente, e velocidade de rosca de $50 \mathrm{rpm}$. Para a obtenção dos filmes biodegradáveis, todos os sistemas foram moldados por compressão em uma prensa hidraúlica MH-08-MN da MH Equipamentos Ltda, sob as seguintes condições: temperatura de $180^{\circ} \mathrm{C}$, carga aplicada de $4-4,5$ toneladas e durante 20 segundos.

O teste de biodegradação em solo simulado foi realizado segundo a norma ASTM G $160-03^{[5]}$, por um período de 60 dias. Neste ensaio foram analisados os filmes de PHB puro, os filmes de PHB/Argila Natural (1\%, 3\% e 6\%) e os filmes de PHB/Argila Organofílica (1\%, 3\% e 6\%). Inicialmente, o solo fértil utilizado no ensaio de biodegradação foi preparado durante uma semana com o acompanhamento do $\mathrm{pH}$ e umidade, com o intuito de padronizar as variáveis existentes antes do ensaio de biodegradação, e assim, torná-lo adequado para o início do ensaio.

Os filmes foram confeccionados com dimensões de $50 \mathrm{~mm} \times 50 \mathrm{~mm}$, conforme a norma ASTM G 160-03 e todas as amostras foram pesadas e identificadas com 
o auxílio de fio de naylon e etiquetadas e logo após, enterradas verticalmente para retiradas nos períodos de 15 , 30,45 e 60 dias. Os sistemas foram avaliados em triplicata. Não foram reportados resultados na $4^{\mathrm{a}}$ retirada (60 dias), devido o elevado nível de biodegradação, impossibilitando o manuseio com as amostras e consequentemente, gerando imprecisão das medidas.

No intuito de visualizar as modificações macroscópicas foram registradas imagens dos bionanocompósitos com o auxílio de uma câmera digital Sony Cyber-shot DSC-W350 14.0 Megapixels.

Os sistemas foram colocados em uma estufa a uma temperatura de $30{ }^{\circ} \mathrm{C}\left( \pm 2{ }^{\circ} \mathrm{C}\right)$ e umidade entre 75 a $95 \%$. Os testes de $\mathrm{pH}$ e umidade foram realizados quinzenalmente para controle das condições ambientais e da qualidade do solo para o ensaio. A água perdida durante o ensaio, devido à evaporação, foi reposta semanalmente, com o auxílio de um borrifador.

\subsection{Método intercalação por solução}

Para a preparação dos filmes pelo método intercalação por solução, o PHB foi previamente peneirado e seco em estufa a $70^{\circ} \mathrm{C}$ por 24 horas. Após esse tempo, foi adicionado o solvente e a solução foi agitada para ocorrer o inchamento do polímero. Em seguida, a solução foi submetida à agitação e aquecimento a $80^{\circ} \mathrm{C}$ durante 3 horas. Para reduzir a perda do solvente por vaporização, foi montado um sistema de condensação, através de um condensador modelo Allihn. Para a formação dos filmes de PHB puro e para os filmes dos bionanocompósitos nos percentuais de 1, 3 e $6 \%$ de argila vermiculita modificada ou natural, as referidas soluções foram vertidas em placas de mármores, espalhando-se livremente, e em seguida, os filmes foram retirados das placas.

O teste de biodegradação dos sistemas também foi realizado segundo a norma ASTM G160-03 ${ }^{[5]}$. Os sistemas foram colocados em um ambiente fechado e mantidos a uma temperatura de $24{ }^{\circ} \mathrm{C}\left( \pm 2{ }^{\circ} \mathrm{C}\right)$ e umidade entre 85 e $95 \%$. Os testes de $\mathrm{pH}$ e umidade do solo, foram realizados a cada retirada de amostras para controle das condições do ambiente e do solo para o ensaio. Devido à perda de umidade foi inserido um umidificador, a fim de aumentar a ventilação e circulação de ar no ambiente.

As amostras com dimensões de $50 \times 50 \mathrm{~mm}$ foram pesadas, identificadas e em seguida enterradas/acondicionadas em solo preparado. Ao final de cada retirada as amostras foram limpas e secas a $23^{\circ} \mathrm{C}$. Os sistemas foram acompanhados quanto à perda de massa e aspecto visual durante 24 dias. Esse período foi dividido em três retiradas: 06, 12 e 24 dias.

\subsection{Difração de raios-X (DRX)}

Todos os sistemas antes de serem submetidos aos testes de biodegradação foram caracterizados por difração de raios-X em um difratômetro da marca Shimadzu XDR 6000,

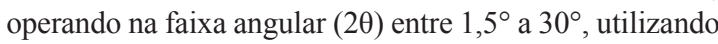
$\mathrm{K} \alpha$ de $\mathrm{Cu}$ como radiação incidente, a tensão realizada foi de $40 \mathrm{Kv}$, corrente $30 \mathrm{~mA}$ e passo $0,02^{\circ}$.A varredura foi realizada na superfície dos filme dos bionancompósitos $(50 \times 50 \mathrm{~mm})$

\section{Resultados e Discussão}

\subsection{Difração de raios-X (DRX): intercalação por fusão e intercalação por solução}

As análises de difração de raios- $X$ foram realizadas na argila organofílica e nos filmes dos bionanocompósitos, a fim de investigar a dispersão das cadeias da argila na matriz polimérica, além de comparar a cristalinidade dos sistemas.

A Figura 1 apresenta os difratogramas da argila vermiculita organofílica (Org) e dos filmes dos bionacompósitos obtidos pela técnica de intercalação por fusão na presença da argila sem modificação (FPHB NAT 1\%, FPHB NAT 3\% e FPHB NAT 6\%), e dos sistemas na presença da argila organofilizada (FPHB ORG 1\%, FPHB ORG 3\% e FPHB ORG 6\%). E a Figura 2 apresenta os difratogramas da argila organofílica (Org) e dos filmes dos bionacompósitos obtidos pela técnica de intercalação por solução na presença da argila sem modificação (SPHB NAT 1\%, SPHB NAT 3\% e SPHB NAT 6\%), e na presença da argila organofilizada (SPHB ORG $1 \%$, SPHB ORG 3\% e SPHB ORG 6\%).

A argila organofílica (ORG) apresentou espaçamentos basais $\left(\mathrm{d}_{001}=45,50 \AA \hat{2}, \mathrm{~d}_{002}=37,72 \AA \mathrm{e} \mathrm{d}_{003}=18,62 \AA\right)$, possivelmente devido a alguma quantidade de argila não intercalada ${ }^{[14]}$. Uma explicação seria que o aparecimento de vários espaçamentos basais durante a intercalação parece

\section{FPHB ORG 6\%}

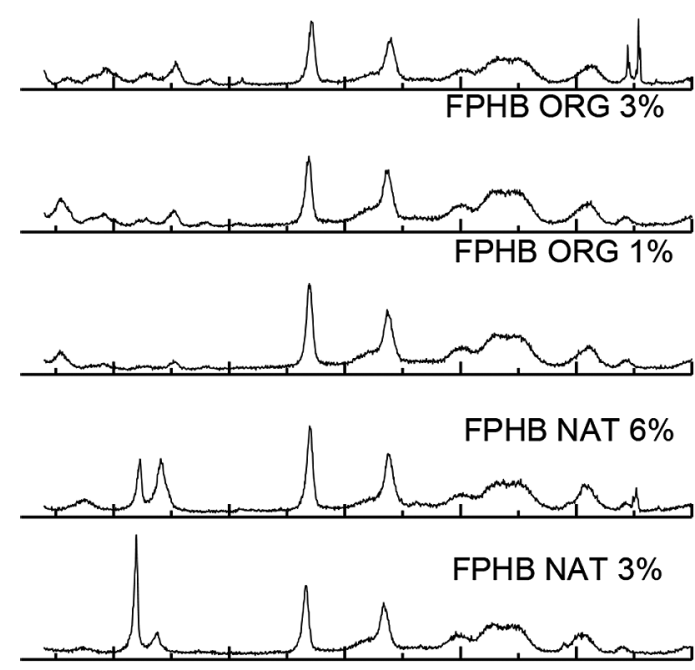

FPHB NAT $1 \%$

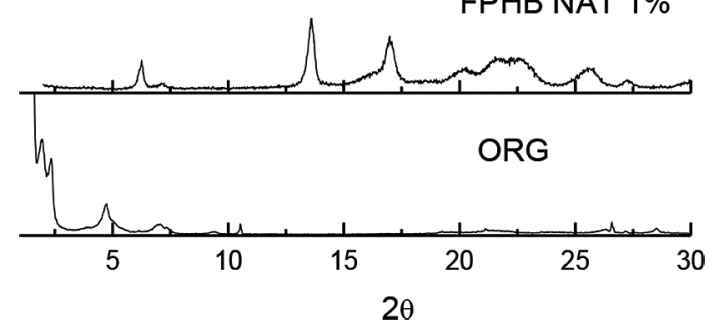

Figura 1. Difratogramas da argila organofílica e dos bionanocompósitos obtidos via intercalação por fusão. 


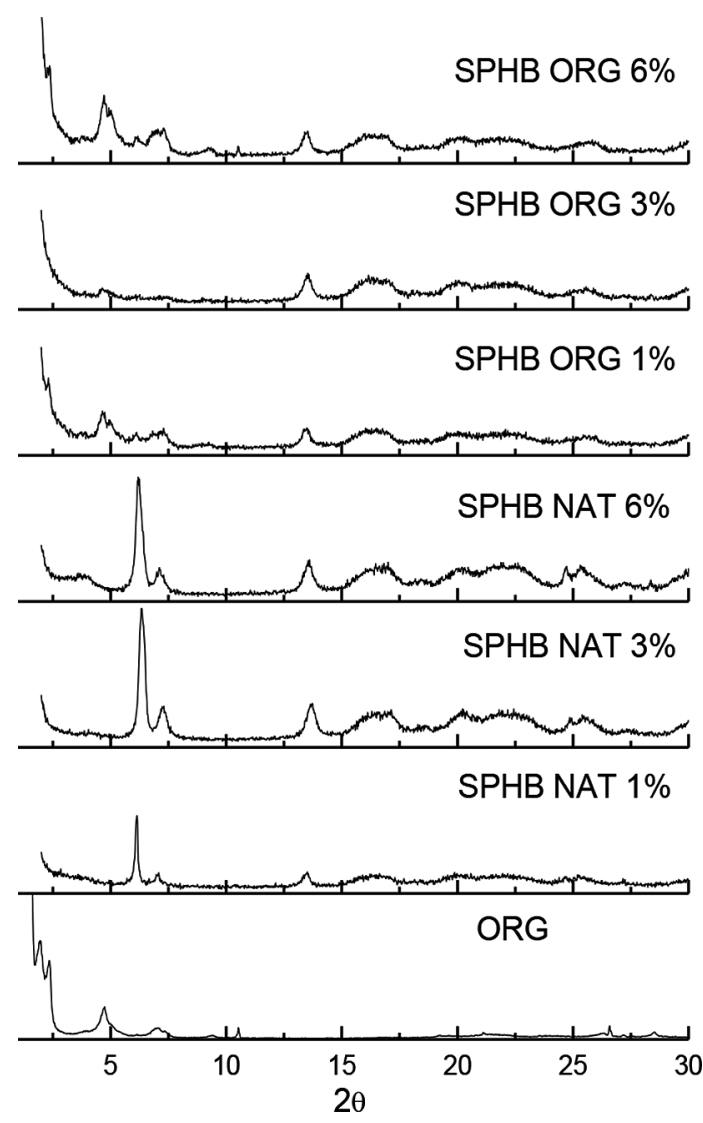

Figura 2. Difratogramas da argila organofílica e dos bionanocompósitos obtidos via intercalação por solução.

está associado à não uniformidade da distribuição dos íons de sódio entre as camadas do argilomineral e a troca seletiva do sódio pelo cátion do sal ${ }^{[15]}$. Isto provocaria a formação de arranjos diferentes do sal ao longo da superfície do material, com suas moléculas formando camadas laterais, simples ou duplas em algumas regiões, e arranjos estendidos parafínicos, em camada simples ou dupla ${ }^{[16]}$.

Os picos de difração referente as distância interplanar basal da argila foram observados na região de baixo ângulo dos difratogramas para os bionanocompósitos via fusão e por via solução. Para os sistemas preparados pela técnica de intercalação por fusão, contendo argila sem modificação, poucas mudanças aconteceram na estrutura interlamelar da argila, indicando aspecto de um microcompósito. Já para os sistemas a base de argila organofílica, aparentemente ocorreu uma maior intercalação do polímero entre as lamelas da argila, uma vez os picos da argila foram deslocados para ângulos menores e consequentemente, aumentando a distância interplanar basal, indicando a formação de prováveis estruturas intercaladas com tendência à esfoliação ${ }^{[17-19]}$.

Para os sistemas preparados via solução com argila sem modificação, também foram observadas mudanças significativas no padrão de difração, independente do teor de carga adicionado. Enquanto que a presença da argila organofilizada reduziu a intensidade dos picos, de maneira até mais intensa quando comparados aos sistemas preparados via fusão. Esse comportamento não era esperado para os sistemas produzidos via intercalação por solução, já que a preparação dos mesmos é provida apenas de uma agitação mecânica pouco agressiva, e consequentemente, refletindo numa baixa dispersão da carga, enquanto os sistemas preparados via fusão era esperado uma melhor dispersão, devido ao maior cisalhamento presente na preparação dos bionanocompósitos.

Os difratogramas dos bionanocompósitos obtidos pela técnica de intercalação por fusão (Figura 1) mostraram que as caracteristicas dos picos de PHB foram mantidas ${ }^{[20,21]}$. Este fato sugere que a estrutura cristalina de PHB não é alterada pela presença das argilas e pelo tipo de processamento ${ }^{[16]}$. Foi observado comportamento diferente para os bionanocompósitos obtidos pela técnica de intercalação por solução (Figura 2), onde os picos referentes ao $\mathrm{PHB}$ apresentaram uma diminuição dos picos ou quase o desaparecimento, fato atribuído a forma de preparo do filme (casting), gerando aspecto mais rugoso e aparência menos homogênea.

\subsection{Inspeção visual: método intercalação por fusão}

A inspeção visual realizou-se por meio de registros de imagens dos filmes após as retiradas, em que é possível verificar as regiões atacadas por fungos e bactérias pela coloração microbiana. Os registros foram analisados antes de entrarem em contato com o solo e ao término de cada período.

Observa-se de uma maneira geral o mesmo perfil de degradação para os filmes preparados pela técnica de intercalação por fusão, independente do tipo de argila (natural ou organofílica). Os sistemas apresentaram manchas esbranquiçadas em toda sua extensão. Resultados semelhantes foram observados por Vanin et al. ${ }^{[22]}$, sendo possível perceber que os microorganismos aderiram à superfície dos filmes biodegradáveis e que as manchas se tornam mais acentuadas com os maiores tempos de exposição. A Figura 3 ilustra o aspecto visual dos sistemas PHB Puro, PHB Nat $1 \%$ e PHB Org $1 \%$ antes do teste, e para a $1^{\mathrm{a}}, 2^{\mathrm{a}}$ e $3^{\mathrm{a}}$ retiradas. É possível observar através das fotografias que os filmes sofreram alterações em sua superfície, conhecidamente segundo Flemming ${ }^{[23]}$, como biodeterioração, que corresponde a um processo interfacial em que os microrganismos atacam e colonizam a superfície do polímero causando modificações superficiais por deposição de material extracelular excretado por eles, acúmulo de água, penetração na matriz com filamentos microbianos, e excreção de pigmentos microbianos lipofílicos que colorem o polímero.

A aparência dos filmes corrobora com os valores de perda de massa que posteriormente serão apresentados, onde verificou-se que os sistemas na presença da argila vermiculita modificada apresentaram menores índices de perda de massa, ou seja, a argila modificada atuou como uma barreira protetora a biodegradação e, consequentemente, apresentando aparência menos desintegrada.

\subsection{Inspeção visual: método intercalação por solução}

A Figura 4 ilustra a aparência dos filmes após a $2^{\circ}$ retirada (índice 2 ) e após a $3^{\circ}$ retirada (índice 3 ), (a) PHB puro, (b, c, d) PHB NAT 1\%, 3\% e 6\% (respectivamente), (e, f, g) PHB ORG 1\%, 3\% e 6\%, respectivamente. Observam-se 


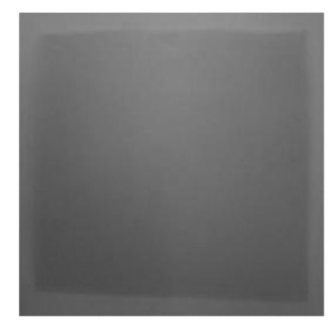

PHB PURO

Antes do teste

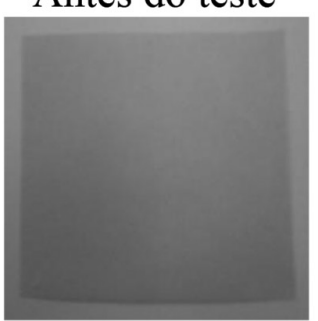

PHB NAT $1 \%$

Antes do teste

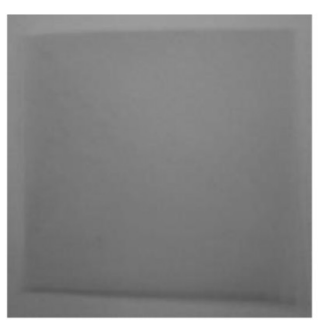

PHB ORG 1\% Antes do teste

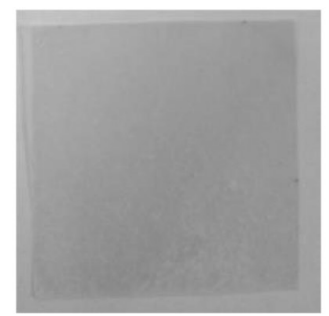

PHB PURO

Após 15 dias

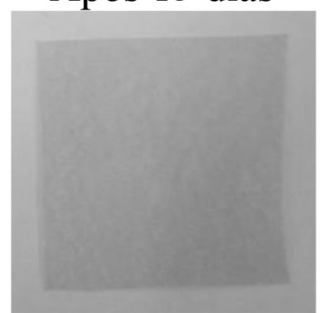

PHB NAT $1 \%$

Após 15 dias

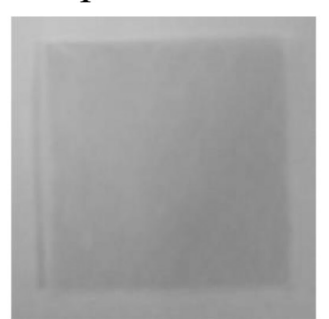

PHB ORG 1\% Após 15 dias

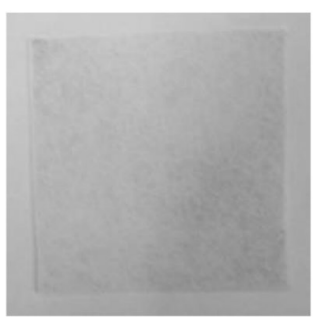

PHB PURO

Após 30 dias
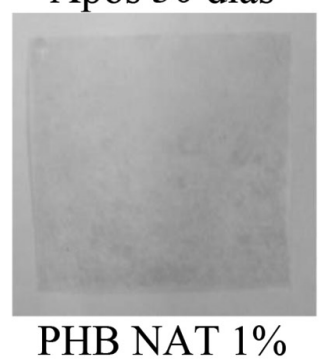

Após 30 dias

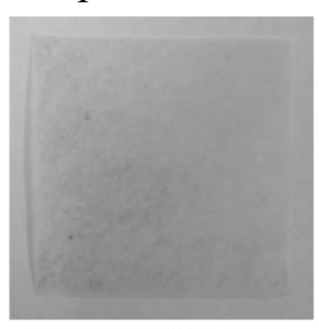

PHB ORG 1\%

Após 30 dias

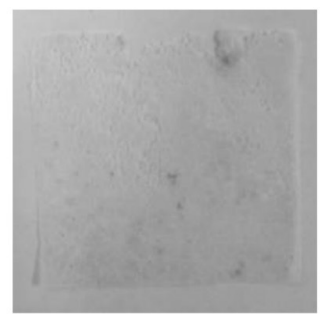

PHB PURO

Após 45 dias

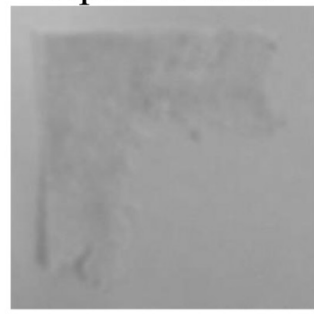

PHB NAT 1\%

Após 45 dias

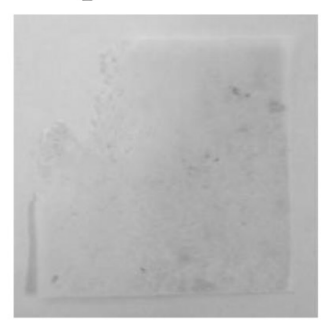

PHB ORG $1 \%$

Após 45 dias

Figura 3. Fotografias dos filmes biodegradáveis PHB Puro, PHB Nat 1\% e PHB Org 1\% em diferentes tempos em contato com o solo.
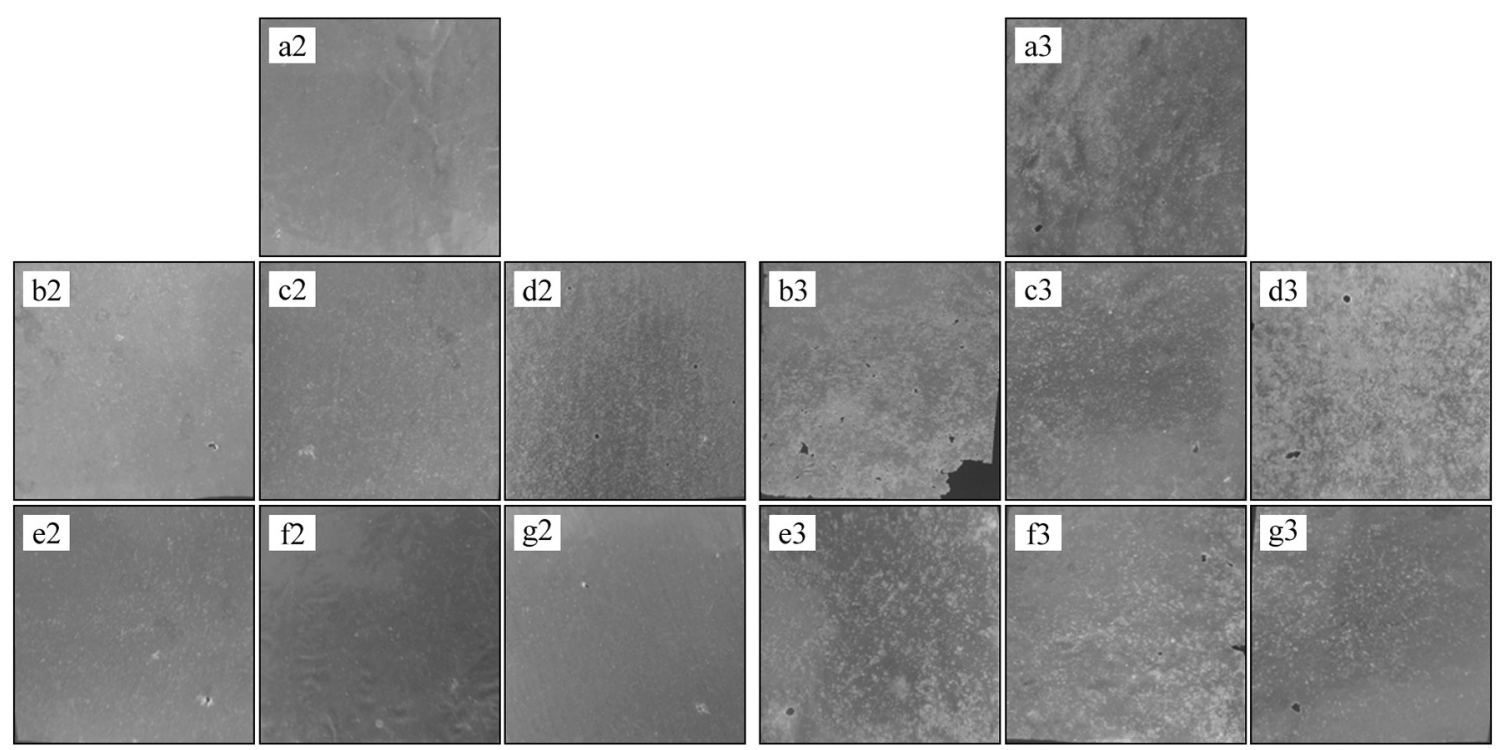

Figura 4. Fotografias dos filmes após a $2^{\circ}$ retirada (índice 2) e após a $3^{\circ}$ retirada (índice 3), (a) PHB puro, (b, c, d) PHB NAT $1 \%, 3 \%$ e $6 \%$ (respectivamente); (e, f, g) PHB ORG 1\%, 3\% e 6\% (respectivamente). 
no aspecto nos filmes na segunda retirada, pouco ataque de microrganismos, estando com aparência quase intacta. É possível que, devido ao pouco tempo (12 dias) e a temperatura de $24{ }^{\circ} \mathrm{C}$, os microrganismos tiveram dificuldades para se desenvolverem. Já para os filmes na terceira retirada podemos observar áreas atacadas por microrganismos, com destaque para $\left(b_{3}\right)$ PHB NAT $1 \%,\left(d_{3}\right)$ PHB NAT $6 \%$ e $\left(f_{3}\right)$ PHB ORG $3 \%$ que foram os mais biodegradados, devido maior tempo de exposição ao solo (24 dias). Esse comportamento corroborá com os resultados de perda de massa que serão apresentandos posteriomente.

Como as regiões amorfas degradam mais rapidamente em relação às regiões cristalinas, podemos afirmar que, com a incorporação dos sais na argila, provavelmente melhorou o empacotamento nas cadeias da argila com PHB, ficando assim menos suscetível aos ataques de microrganismos.

De maneira geral, o aspecto visual dos filmes obtidos pelos dois métodos apresentou o mesmo perfil de ataque, independente da concentração de argila, apresentando manchas esbranquiçadas em toda sua extensão. E ainda, ocorreu o aparecimento de manchas escuras em alguns filmes, comprovando a presença dos microrganismos após a realização do ensaio de biodegradação.

\subsection{Perda de massa: método intercalação por fusão}

A Tabela 1 e a Figura 5 apresentam os valores médios para a perda de massa dos filmes biodegradáveis de PHB Puro, PHB Nat $1 \%$ e PHB Org $1 \%$ na $1^{\mathrm{a}}, 2^{\mathrm{a}}$ e $3^{\mathrm{a}}$ retiradas, correspondendo a 15, 30 e 45 dias de exposição ao solo.

Observa-se que conforme aumenta o tempo em contato com o solo a quantidade de perda de massa do material é maior. Resultados semelhantes foram observados por Casarin et al. ${ }^{[2]}$. Para 45 dias de exposição, o sistema PHB Nat $1 \%$ apresentou maior perda de massa $89,75 \%$, enquanto

Tabela 1. Perda de massa dos filmes biodegradáveis PHB Puro, PHB Nat $1 \%$ e PHB Org $1 \%$ para diferentes tempos em contato com o solo.

\begin{tabular}{lccc}
\multicolumn{1}{c}{ Amostras } & 15 dias (\%) & 30 dias (\%) & 45 dias (\%) \\
\hline PHB PURO & 1,48 & 24,00 & 48,98 \\
PHB NAT 1\% & 3,46 & 34,60 & 89,75 \\
PHB ORG 1\% & 1,34 & 11,25 & 42,57 \\
\hline
\end{tabular}

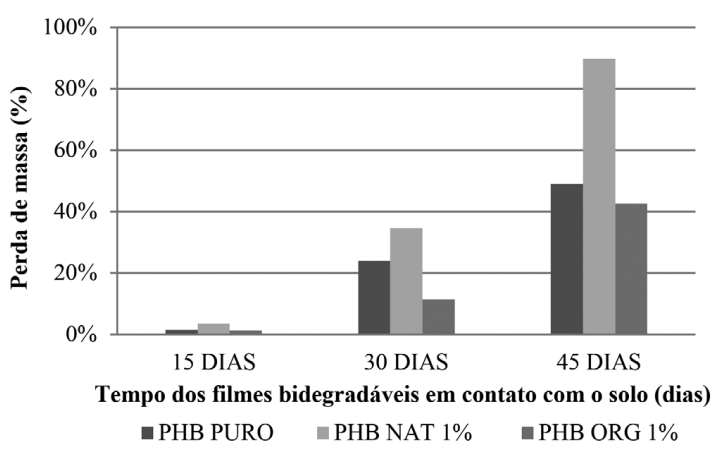

Figura 5. Percentual da perda de massa dos filmes biodegradáveis PHB Puro, PHB Nat $1 \%$ e PHB Org 1\% para diferentes tempos em contato com o solo. o PHB Puro e o PHB Org 1\% apresentaram perda de massa de $48,98 \%$ e $42,57 \%$, respectivamente.

A Tabela 2 e a Figura 6 apresentam os valores médios para a perda de massa dos filmes biodegradáveis PHB Puro, PHB Nat 3\% e PHB Org 3\% para os mesmos períodos de retiradas mencionados anteriormente. Verifica-se que o $\mathrm{PHB}$ Nat 3\% apresentou maior perda de massa com $60,89 \%$, enquanto o PHB Puro e o PHB Org 3\% apresentaram perda de massa de $48,98 \%$ e $24,99 \%$, respectivamente, para os 45 dias de exposição.

A Tabela 3 e a Figura 7 apresentam os valores médios para a perda de massa dos filmes biodegradáveis PHB Puro, PHB Nat 6\% e PHB Org 6\% para os mesmos períodos de retiradas. Percebe-se que o PHB Org 6\% apresentou maior perda de massa, para a $1^{\mathrm{a}}$ e $2^{\mathrm{a}}$ retirada, enquanto o PHB Puro e o PHB Nat $6 \%$ apresentaram perda de massa de $48,98 \%$ e $46,36 \%$, respectivamente para 45 dias de exposição ao solo simulado.

Observa-se que os filmes obtidos pelo método intercalação por fusão, na presença e na variação do percentual da argila organofílica, levaram a menores índices de perda de massa, ou seja a argila modificada atuou como uma barreira protetora a biodegradação. A argila organofílica tem duas funções opostas na estabilidade dos bionanocompósitos polímero/argila: uma, é o efeito de barreira exercido pela argila, que pode melhorar a estabilidade a biodegradação e a outra, é o efeito catalítico da argila que pode acarretar a degradação da matriz polimérica diminuindo a estabilidade. Quando se adicionou pequena fração de argila organofílica na matriz polimérica a dispersão foi favorecida, mas com a adição de níveis elevados dessa argila, o efeito catalítico é predominante e a estabilidade do bionanocompósito foi diminuída $^{[25]}$. Este mesmo comportamento foi observado para o sistema PHB ORG 6\%.

Tabela 2. Percentual da perda de massa dos filmes biodegradáveis PHB Puro, PHB Nat 3\% e PHB Org 3\% em diferentes tempos em contato com o solo.

\begin{tabular}{lccc}
\hline \multicolumn{1}{c}{ Amostras } & 15 dias (\%) & 30 dias (\%) & 45 dias (\%) \\
\hline PHB PURO & 1,48 & 24,00 & 48,98 \\
PHB NAT 3\% & 6,21 & 20,48 & 60,89 \\
PHB ORG 3\% & 1,16 & 20,70 & 24,99 \\
\hline
\end{tabular}

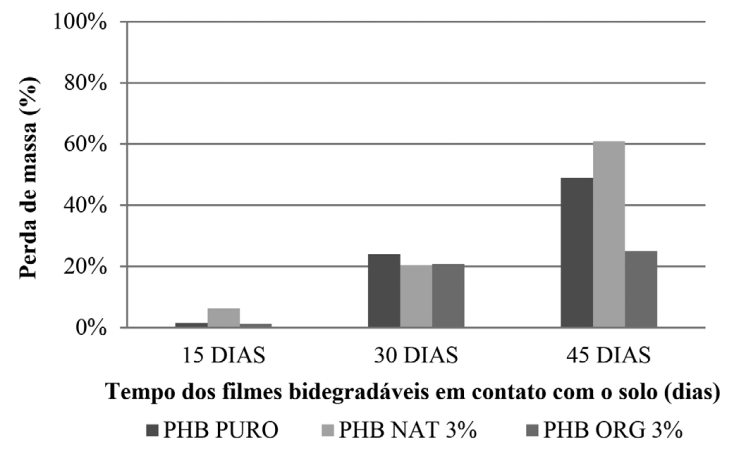

Figura 6. Percentual da perda de massa dos filmes biodegradáveis PHB Puro, PHB Nat 3\% e PHB Org 3\% em diferentes tempos em contato com o solo. 
Tabela 3. Percentual da perda de massa dos filmes biodegradáveis PHB Puro, PHB Nat 6\% e PHB Org 6\% em diferentes tempos em contato com o solo.

\begin{tabular}{lccc}
\hline \multicolumn{1}{c}{ Amostras } & 15 dias (\%) & 30 dias (\%) & 45 dias (\%) \\
\hline PHB PURO & 1,48 & 24,00 & 48,98 \\
PHB NAT 6\% & 1,20 & 11,25 & 46,36 \\
PHB ORG 6\% & 5,44 & 37,22 & 40,46 \\
\hline
\end{tabular}

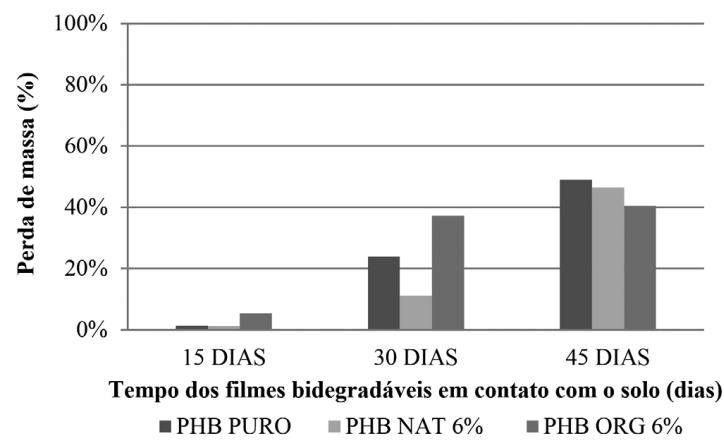

Figura 7. Variação percentual da perda de massa dos filmes biodegradáveis PHB Puro, PHB Nat 6\% e PHB Org 6\% em diferentes tempos em contato com o solo.

Outra hipótese, é que durante a fase inicial de desintegração, as cadeias de alto peso molecular de PHB são hidrolisadas formando cadeias de baixo peso molecular. Esta reação pode ser retardada em sistemas contendo argilas organofílicas, que impedem o movimento e a difusão na maior parte do filme ${ }^{[26]}$. Já para os sistemas contendo argilas não modificadas, foi observada uma maior perda de massa, este fato é explicado devido à presença de sítios ácidos de Lewis nas camadas da argila inorgânica, ou pela presença de água residual e a própria natureza hidrofílica, assim favorecendo a biodegradação ${ }^{[16]}$.

Os maiores percentuais de perda de massa observados para os sistemas na presença de argila organofílica podem também estar relacionado ao baixo potencial de ação fungicida no sal quaternário de amônio presente na estrutura da argila vermiculita. Os compostos quaternário de amônio são agentes tensoativos catiônicos com boa atividade germicida ${ }^{[27]}$.

\subsection{Perda de massa: método intercalação por solução}

A Tabela 4 e a Figura 8 apresentam os valores médios para a perda de massa dos filmes biodegradáveis de PHB Puro, PHB Nat $1 \%$ e PHB Org $1 \%$ na $1^{\mathrm{a}}, 2^{\mathrm{a}}$ e $3^{\mathrm{a}}$ retiradas, correspondendo a 06, 12 e 24 dias de exposição ao solo.

Observa-se o sistema PHB Nat 1\% para 24 dias de exposição apresentou maior perda de massa $19,26 \%$, enquanto o PHB Puro e o PHB Org $1 \%$ apresentaram perda de massa de $10,92 \%$ e $6,62 \%$, respectivamente.

A Tabela 5 e a Figura 9 apresentam os valores médios para a perda de massa dos filmes biodegradáveis PHB Puro, PHB Nat 3\% e PHB Org 3\% para os mesmos períodos de retiradas mencionados anteriormente. Verifica-se que o PHB Org $3 \%$ apresentou maior perda de massa com $16,23 \%$,
Tabela 4. Perda de massa dos filmes biodegradáveis PHB Puro, PHB Nat $1 \%$ e PHB Org 1\% para diferentes tempos em contato com o solo.

\begin{tabular}{lccc}
\hline \multicolumn{1}{c}{ Amostras } & 06 dias (\%) & 12 dias (\%) & 24 dias (\%) \\
\hline PHB PURO & 0,98 & 1,07 & 10,92 \\
PHB NAT 1\% & 0,19 & 1,55 & 19,26 \\
PHB ORG 1\% & 0,23 & 0,94 & 6,62 \\
\hline
\end{tabular}

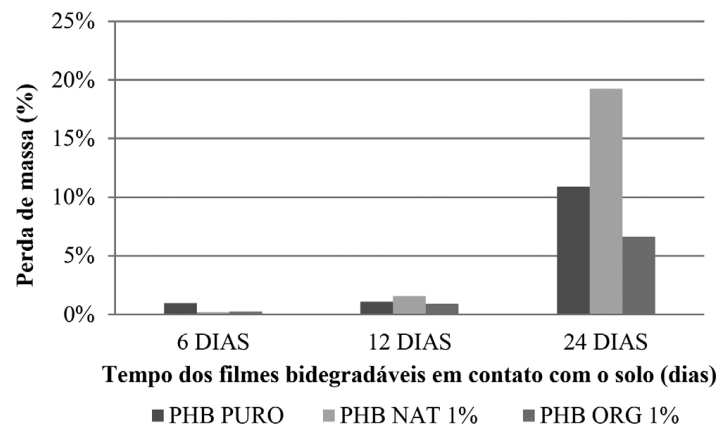

Figura 8. Percentual da perda de massa dos filmes biodegradáveis PHB Puro, PHB Nat $1 \%$ e PHB Org $1 \%$ para diferentes tempos em contato com o solo.

Tabela 5. Perda de massa dos filmes biodegradáveis PHB Puro, PHB Nat 3\% e PHB Org 3\% para diferentes tempos em contato com o solo.

\begin{tabular}{lccc}
\multicolumn{1}{c}{ Amostras } & 06 dias (\%) & 12 dias (\%) & 24 dias (\%) \\
\hline PHB PURO & 0,98 & 1,07 & 10,92 \\
PHB NAT 3\% & 0,07 & 1,22 & 3,88 \\
PHB ORG 3\% & 0,46 & 0,81 & 16,23 \\
\hline
\end{tabular}

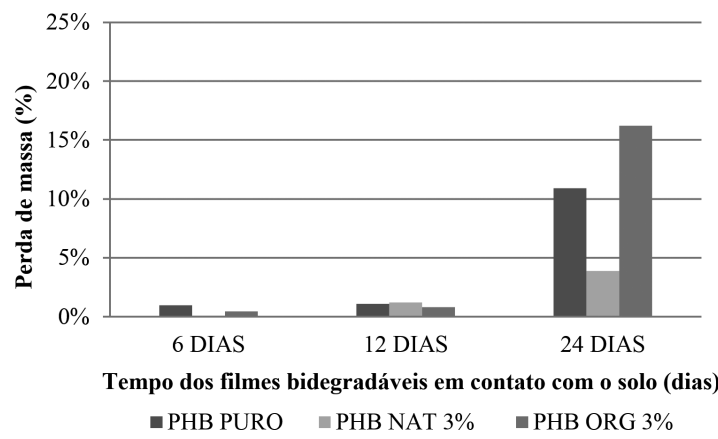

Figura 9. Percentual da perda de massa dos filmes biodegradáveis PHB Puro, PHB Nat 3\% e PHB Org 3\% para diferentes tempos em contato com o solo.

enquanto o PHB Puro e o PHB Nat 3\% apresentaram perda de massa de $10,92 \%$ e $3,88 \%$, respectivamente.

A Tabela 6 e a Figura 10 apresentam os valores médios para a perda de massa dos filmes biodegradáveis PHB Puro, PHB Nat 6\% e PHB Org 6\% para os mesmos períodos de retiradas. Percebe-se que o PHB Nat 6\% apresentou maior perda de massa com 23,33\%, enquanto o PHB Puro e o PHB Org $6 \%$ apresentaram perda de massa de $10,92 \%$ e $2,26 \%$, respectivamente para 24 dias de exposição ao solo simulado. 
Tabela 6. Perda de massa dos filmes biodegradáveis PHB Puro, PHB Nat $6 \%$ e PHB Org 6\% para diferentes tempos em contato com o solo.

\begin{tabular}{lccc}
\multicolumn{1}{c}{ Amostras } & 06 dias (\%) & 12 dias (\%) & 24 dias (\%) \\
\hline PHB PURO & 0,98 & 1,07 & 10,92 \\
PHB NAT 6\% & 0,10 & 3,77 & 22,33 \\
PHB ORG 6\% & 0,12 & 0,42 & 2,26 \\
\hline
\end{tabular}

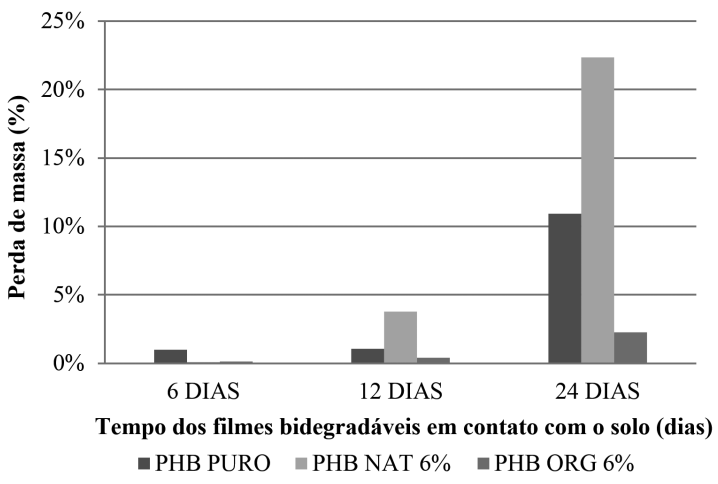

Figura 10. Percentual da perda de massa dos filmes biodegradáveis PHB Puro, PHB Nat 6\% e PHB Org 6\% para diferentes tempos em contato com o solo.

Para os sistemas obtidos pelo método via intercalação por solução, foi observado o mesmo comportamento dos filmes obtidos via fusão, ou seja, a presença e a variação do percentual da argila organofílica, promoveram a índices menores de perda de massa, ou seja a argila modificada também atuou como uma barreira protetora a biodegradação e ação fungicida. Com exceção do sistema com $3 \%$ de argila organofílica.

\section{Conclusões}

Neste trabalho foi analisada a biodegradação dos filmes PHB Puro, e dos sistemas com 1, 3 e $6 \%$ de argila natural e modificada. A diferença nas estruturas dos bionanocompósitos é influenciada pelo método de obtenção, via intercalação por fusão ou solução. Os sistemas foram avaliados de acordo com a norma ASTM G 160-03. Foi observado para todas as amostras, conforme aumenta o tempo em contato com o solo, aumenta também a quantidade de perda de massa do material. O ensaio de biodegradação mostrou-se viável, visto que é um processo adequado, ou seja, os microrganismos estão em ambiente propício para seu desenvolvimento e nutrição.

Nos resultados obtidos pelo método intercalação por fusão, o filme biodegradável PHB Nat $1 \%$ apresentou a maior perda de massa, com $89,75 \%$, em relação aos outros filmes submetidos ao ensaio de biodegradação. Assim, observa-se que o percentual de argila vermiculita natural e os períodos de retiradas influenciaram na deterioração do material. Pelo método intercação por solução, podemos afirmar que as composições de PHB com vermiculita natural foram as mais atacadas por microrganismos, com destaque para a composição PHB Nat 6\%. Já para os filmes de vermiculita organofílica ficaram pouco evidentes os ataques de microrganismos.

\section{Agradecimentos}

Os autores agradecem a FAPEPI e ao CNPq pelo apoio financeiro para a realização deste trabalho. Ao Laboratório Interdisciplinar de Materiais Avançados/CCN/UFPI e a UFCG pela concessão do espaço para a realização do procedimento experimental e a PHB industrial pela doação da matriz polimérica.

\section{Referências}

1. Souza, C. O., Silva, T. L., \& Druzian, J. I. (2012). Estudo comparativo da caracterização de filmes biodegradáveis de amido de mandioca contendo polpas de manga e de acerola. Quimica Nova, 35(2), 262-267. http://dx.doi.org/10.1590/ S0100-40422012000200006.

2. Arrieta, M. P., Fortunati, E., Dominici, F., Rayón, E., López, J., \& Kenny, J. M. (2014). PLA-PHB/cellulose based films: mechanical, barrier and disintegration proper-ties. Polymer Degradation \& Stability, 107, 139-149. http://dx.doi.org/10.1016/j. polymdegradstab.2014.05.010.

3. Arrieta, M. P., Fortunati, E., Dominici, F., López, J., \& Kenny, J. M. (2015). Bionanocomposite films based on plasticized PLA-PHB/cellulose nanocrystal blends. Carbohydrate Polymers, 121, 265-275. http://dx.doi.org/10.1016/j.carbpol.2014.12.056. PMid:25659698.

4. Rosa, D. S., Chui, Q. S. H., Pantano Filho, R., \& Agnelli, J. A. M. (2002). Avaliação da biodegradação de poli- $\beta$-(Hidroxibutirato), poli- $\beta$-(Hidroxibutirato-co-valerato) e poli- $\varepsilon$-(caprolactona) em solo compostado. Polímeros: Ciência e Tecnologia, 12(4), 311317. http://dx.doi.org/10.1590/S0104-14282002000400015.

5. American Society for Testing and Materials - ASTM. (2004). ASTM G160-03: standard pratice for evaluating microbial susceptibility of nonmetallic materials by laboratory soil burial (Vol. 14.04). West Conshohocken: ASTM.

6. Medeiros, I. L., Maracacini, J. M. S. A., Duarte, M. A. T., Pezzin, S. H., Sobral, J. C., Balzer, P. S., Bernardo, H. P., Everling, M. T., \& Pezzin, A. P. T. (2005). Inovação em materiais: estudo para desenvolvimento de escova dental a partir de polímeros biodegradáveis. Revista Matéria, 10(1), 1-7. Recuperado em 20 de novembro de 2014, de http://www.materia.coppe.ufrj. br/sarra/artigos/artigo 10642

7. Quental, A. C., Carvalho, F. P., Tada, E. S., \& Felisberti, M. I. (2010). Blendas de PHB e seus copolímeros: miscibilidade e compatibilidade. Quimica Nova, 33(2), 438-446. http://dx.doi. org/10.1590/S0100-40422010000200035.

8. Coelho, N. S., Almeida, Y. M. B., \& Vinhas, G. M. (2008). A biodegradabilidade da blenda de poli( $\beta$-hidroxibutirato-covalerato)/amido anfótero na presença de microorganismos. Polimeros: Ciência e Tecnologia, 18(3), 270-276. http://dx.doi. org/10.1590/S0104-14282008000300014.

9. Gomes, E. V. D., Visconte, L. L. Y., \& Pacheco, E. B. A. V. (2010). Processo de organofilização de vermiculita brasileira com cloreto de cetiltrimetilamônio. Cerâmica, 56(337), 44-48. http://dx.doi.org/10.1590/S0366-69132010000100008.

10. Souza, M. A., Pessan, L. A., \& Rodolfo, A., Jr. (2006). Nanocompósitos de poli(cloreto de vinila)(PVC)/argilas organofílicas. Polímeros: Ciência e Tecnologia, 16(4), 257262. http://dx.doi.org/10.1590/S0104-14282006000400003.

11. Rodrigo, E. J. R., Nascimento, S. A. M., Tavares, M. I. B., \& Merat, P. P. (2012). Investigação da influência do processamento na dinâmica molecular de nanocompósitos de policarbonato e 
argila organofílica obtidos via intercalação por fusão. Polímeros: Ciência e Tecnologia, 22(5), 436-439. http://dx.doi.org/10.1590/ S0104-14282012005000058.

12. Barbosa, R., Araújo, E. M., Oliveira, A. D., \& Mélo, T. J. A. (2006). Efeito de sais quaternários de amônio na organofilização de uma argila bentonita nacional. Cerâmica, 52(324), 264-268. http://dx.doi.org/10.1590/S0366-69132006000400009.

13. Mesquita, P. J. P. (2014). Avaliação da biodegradação e caracterização térmica e estrutural de blendas e de bionanocompósitos PHB/PP-g-MA/argila (Dissertação de mestrado). Universidade Federal do Piauí, Teresina.

14. Araújo, E. M., Barbosa, R., Oliveira, A. D., Morais, C. R. S., Souza, A. G., \& Melo, T. J.A. (2007). Thermal and mechanical properties of PE/organoclay nanocomposites. Journal of Thermal Analysis and Calorimetry, 87(3), 811-814. http:// dx.doi.org/10.1007/s10973-006-7758-0.

15. Lee, S. Y., \& Kim, S. J. (2002). Delamination behavior of silicate layers by adsorption of cationic surfactants. Journal of Colloid and Interface Science, 248(2), 231-238. http://dx.doi. org/10.1006/jcis.2002.8222. PMid:16290526.

16. Puglia, D., Fortunati, E., D’Amico, D. A., Manfredi, L. B., Cyras, V. P., \& Kenny, J. M. (2014). Influence of organically modified clays on the properties and disintegrability in compost of solution cast poly(3-hydroxybutyrate) films. Polymer Degradation \& Stability, 99, 127-135. http://dx.doi. org/10.1016/j.polymdegradstab.2013.11.013.

17. Pantani, R., \& Sorrentino, A. (2013). Influence of crystallinity on the biodegradation rate of injection-moulded poly (lactic acid) samples in controlled composting conditions. Polymer Degradation \& Stability, 98(5), 1089-1096. http://dx.doi. org/10.1016/j.polymdegradstab.2013.01.005.

18. Salehabadi, A., \& Bakar, M. A. (2012). Poly (3-hydroxybutyrate) organo modified montmorillonite nano hybrid; preparation and characterization. Advanced Materials Research, 622623, 263-270. http://dx.doi.org/10.4028/www.scientific.net/ AMR.622-623.263.

19. Thiré, R. M. S. M., Arruda, L. C., \& Barreto, L. S. (2011). Morphology and thermal properties of poly(3-hydroxybutyrateco-3-hydroxyvalerate)/attapulgite nanocomposites. Materials Research, 14(3), 340-344. http://dx.doi.org/10.1590/S151614392011005000046
20. Chiu, H. J. (2005). Segregation morphology of poly(3hydroxybutyrate)/poly(vinyl acetate) and poly(3-hydroxybutyrateco-10\% 3-hydroxyvalerate)/poly(vinyl acetate) blends as studied via small angle x-ray scattering. Polymer, 46(11), 3906-3913. http://dx.doi.org/10.1016/j.polymer.2005.03.032.

21. Hurrell, B. L., \& Cameron, R. E. (1998). A wide-angle x-ray scattering study of the ageing of poly(hydroxybutyrate). Journal of Materials Science, 33(7), 1709-1713. http://dx.doi. org/10.1023/A:1004364112658.

22. Vanin, M., Santana, C. C., Torriani, I. L., Privelic, T., \& Duek, E. A. R. (2004). Estudo da degradação "in vitro" de blendas de poli( $\beta$-Hidroxibutirato) (PHB)/ poli(L-ácido latico) (PLLA) na forma de filmes. Polímeros: Ciência e Tecnologia, 14(3), 187-193. http://dx.doi.org/10.1590/S0104-14282004000300015.

23. Flemming, H. C. (1998). Relevance of biofilms for the biodeterioration of surfaces of polymeric materials. Polymer Degradation \& Stability, 59(1-3), 309-315. http://dx.doi. org/10.1016/S0141-3910(97)00189-4.

24. Casarin, S. A., Agnelli, J. A. M., Malmonge, S. M., \& Rosário, F. (2013). Blendas PHB/copoliésteres biodegradáveis: biodegradação em solo. Polímeros: Ciência e Tecnologia, 23(1), 115-122. http://dx.doi.org/10.1590/S0104-14282013005000003.

25. Zhao, C., Qin, H., Gong, F., Menh, F., Zhang, S., \& Yang, M. (2005). Mechanical, thermal and flammability properties of polyethylene/clay nanocomposites. Polymer Degradation \& Stability, 87(1), 183-189. http://dx.doi.org/10.1016/j. polymdegradstab.2004.08.005.

26. Wang, S., Song, C., Cheng, G. T., Guo, T., Liu, J., Zhang, B., \& Takeuchi, S. (2005). Characteristics and biodegradation properties of poly(3-hydroxybutyrate-co-3-hydroxyvalerate)/ organophilic montmorillonite (phbv/ommt) nanocomposite. Polymer Degradation \& Stability, 87(1), 69-76. http://dx.doi. org/10.1016/j.polymdegradstab.2004.07.008.

27. Germano, P. M. L., \& Germano, M. I. S. (2001). Higiene e vigilância sanitária de alimentos. São Paulo: Varela. 629 p.

Enviado: Dez. 14, 2014

Revisado: Jan. 13, 2015 Aceito: Maio 04, 2015 